would frequently give the attending physician valuable information regarding the source of the causative infections and personal habits which might have bearing on the disease.

This brief outline of a very important as well as interesting subject will, it is hoped, serve as a stimulus to others for making additional observations and investigations along the lines indicated.

\section{THE PSEUDOREACTION IN THE SCHICK TEST AND ITS CONTROL*}

\section{ABRAHAM ZINGHER, M.D. NEW YORK}

The pseudoreaction in the Schick test is caused, not by the soluble diphtheria toxin, but by the protein substance of the diphtheria bacillus which is present in tlie solution used for the test. The reaction may be obtained in individuals who have enough natural antitoxin to render them immune to diphtheria. It is important, therefore, to control these pseudoreactions, which are quite frequent in the adult, especially women (from 20 to 30 per cent.), but relatively rare in children. These control tests may be made after the reaction with the regular Schick test has developed, or, especially in the adult, the two tests may be made simultaneously, one on each forearm, and the clinical course of the reactions thus compared.

For the Schick test, the bureau of laboratories of the health department is supplying an outfit which contains carefully measured amounts of undiluted diphtheria toxin. This outfit, as previously described by me, ${ }^{1}$ consists of a capillary tube which contains a little over one minimum lethal dose of a ripened diphtheria toxin, a small rubber bulb for expelling the toxin, and a 10 c.c. bottle of normal saline solution for diluting the toxin. Every 0.2 c.c. of the dilution represents one fiftieth minimum lethal dose, the amount used in the Schick test.

The outfit for controlling the positive, pseudo and combined reactions is similar to the Schick test outfit, but the toxin in the capillary tube has been heated to $75 \mathrm{C}$. $(167 \mathrm{~F}$.) for five minutes. Heating the toxin at this temperature destroys the soluble diphtheria toxin, but does not appreciably affect the protein of the diphtheria bacillus.

We made an attempt during the past two and onehalf years, during which time the work with the Schick test has been carried on by us at the Willard J'arker Hospital and various other institutions, to conirol as many of the pseudo and combined reactions as possible. Convinced of the fact that the pseudoreaction was a local anaphylactic response to the protein of the diphtheria bacillus, we at first used a solution containing the autolyzed substance of the KlebsLoeffler bacillus. This autolysate consisted of a fortycight hour agar growth of the diphtheria bacillus (No. 8 Park \& Williams) which was suspended in salt solution in the proportion of 10 c.c. of saline to a Blake bottle culture. The bacterial suspension was washed four times with saline solution, made up to its original volume with distilled water, then shaken

* From the Research Laboratory of the Department of Health, (ity of New York.

1. Zingher, Abraham: A Simple Outfit for the Distribution of Toxin for the Schick Test, THE Journal A. M. A., July 24, 1915 [. 329. for two hours, killed at $57 \mathrm{C}$. (134.6 F.) for fortyfive minutes and allowed to autolyze in the thermostat over night. The solution was then passed through a Berkefeld filter, and the filtrate considered as a concentrated autolysate. Ten c.c. of the autolysate, representing the growth of one Blake bottle, injected subcutaneously into a guinea-pig, did not show any evidence of soluble toxin. When the bacterial suspension was injected before its passage through a Berkefelil filter, a small knoblike induration developed, which soon disappeared. For use in the control of the Schick test in human beings, the concentrated autolysate was diluted $1: 20$ with salt solution, and 0.2 c.c. injected intradermally. Typical false reactions were obtaincd in the pseudo and combined Schick cases, but none in the negative or positive cases. By using a more concentrated solution of the autolysate, faint reactions could be obtained even in individuals who gave a negative Schick reaction.

The difficulty of estimating the amount of bacillus protein in the diphtheria toxin broth used in the Schick test, which would serve as a guide in making a proper dilution of the autolysate, prompted us finally to abandon the autolysate as a suitable control, and us: either a toxin which had been slightly overneutralized with antitoxin, or a heated toxin in a dilution similar to that used in the Schick test. In the overneutralized toxin ( 2 units of antitoxin to each $\mathrm{L}+$ dose of toxin) we had added, however, another element, the antitoxin. This, though in extreme dilution ( 1 part to a million as finally injected), might yet be objected to as a possible additional source of error. We finally used the heated toxin as representing probably the most accurate way for obtaining a proper control test. A dilution of the protein substance of the diphtheria bacillus is thus obtained which is exactly similar to that in the solution used for the Schick test. The capillary tubes in the outfits represent simply a convenient and accurate way for heating the carefully measured amounts of diphtheria toxin.

The Research Laboratory of the New York City Health Department is now supplying these outfits. They will remain good for use for a long time, since there is no danger of deterioration of an unstable toxin.

The following are the directions accompanying the outfits :

\section{DIRECTIONS FOR USING IIEATED DIPHTHIERIA TOXIN} IN CONTROLLING TIIE SCIIICK REACTION

Break off one end of the capillary tube, push the broken end carefully through the neck of the rubber bullh until it punctures the diaphragm within, and enters the cavity of the bulb; then break off the other end of the tube. Hold the bull, between the thumb and middle finger, place the index fingor over the opening in the larger cnd of the bulb and expel the toxin into the saline. Rinse out the capillary tube by drawing up saline several times, then cork the bottle and shake the diluted toxin. Inject 0.2 c.c. intracutaneously on the flexor surface of the forearm or arm. Observe the reaction at the site of injection at the end of twenty-four, forty-eight, seventy-two and ninety-six hours, and compare with the original Schick reaction.

The capillary tube in this outfit contains the culture fluid similar to that used in the Schick test, but heated to $75 \mathrm{C}$. for five minutes. The heating destroys the diphtheria toxin, which is the active agent in the positive Schick reaction, but does not affect the protein substance of the diphtheria bacillus, which produces the pseudoreaction.

In an individual who gave a positive reaction only, the control test with the heated toxin will be negative. In one 
who gave a pscudoreaction, the control reaction will be of about the same size and appearance, and pass through the same clinical course as the original reaction; e. g., the two reactions showing a similar central area of redness of varying size, surrounded by a secondary areola which shades off into the surrounding skin. Both reactions begin to appear in about six to eighteen hours, reach their height in twentyfour to thirty-six hours and disappear in three to four days, leaving a poorly defined area of pigmentation and generally no scaling.

In an individual who gave a combined reaction in the original test, both the positive and the pseudoreaction may be detected. The positive reaction becomes manifest in the original test at the end of three to four days, when the pseudoeicment of the reaction has disappeared, as a definite circumscribed area of redness, which measures from 1 to 2.5 $\mathrm{cm}$. in diameter and shows superficial scaling with beginning brownish pigmentation. The pseudoreaction is detected in the control test with the heated toxin; the reaction will not bc as marked as in the original Schick test, but passes through the typical clinical course of a false reaction.

\section{FRACTURE OF THE GREATER CORNU OF THE HYOID BONE RESULTING FROM MUSCULAR ACTION*}

\section{H. P. Ashe, M.D., Pittsburgh}

Fractures of the hyoid bone due to muscular action are very rare. Stimson' mentions only two such cases out of forty-five hyoid fractures collected by Malgaigne, Hamilton, Gibb, Gurlt and himself.

The following case of fracture of the left greater cornu of the hyoid bone, having resulted from muscular action, will consequently be of interest:

Miss E. H., aged 29, domestic by occupation, called on me, Feb. 18, 1916, complaining of pain and swelling in the neck, and particularly of change of voice, the occasional absence of which so alarmed her as to be the incentive toward seeking medical aid.

The week previously, February 11, while engaged in the task of arranging portières, she inclined her head forcibly backward, and at the time felt and heard, as she expressed it, "something break in the neck." Swelling of the neck anteriorly, localized pain in the neck produced by motions of the head or tongue, bloody sputum, deglutition embarrassed by frequent choking, and alteration of voice variously muffled, hoarse, or absent were the symptoms early manifested. Cough and dyspnea were not experienced.

The patient was a tall, healthy looking, well developed muscular young woman, whose past medical history was uneventful, barring a tonsillectomy and an appendicectomy undergone a few years previously, since which time she has enjoyed excellent health.

A very discernible, well defined swelling, without ecchymosis, occupied the anterior and anterolateral cervical regions, beginning sharply at the superior border of the hyoid bone, and continuing into and traversing the submental region as far as the inferior border of the inferior maxillary bone.

Emphysema was not present. The hyoid bone was well outlined on palpation, disclosing no perceptible irregularity, save a slight inward deviation of the left greater cornu. Pain and tenderness were elicited when pressure was made at a point on the bone near the juncture of its left greater cornu with its body. Slightly to the left of this point was a slightly enlarged, tender submaxillary lymphatic gland.' Examination of the floor of the mouth and oropharynx proved negative.

As a week had elapsed since the occurrence of the accident, and as union takes place promptly in hyoid fractures, crepitus and preternatural mobility were not present.

* Read before the Allegheny County Medical Society, March 21, 1916.

1. Stimson: Practical Treatise on Fractures and Dislocations, Ed. 6 , Pliludelphia, Lea \& Febiger, 1910, p. 180.
The contours and external surfaces of the thyroid and cricoid cartilages were unaltered, and both could be freely manipulated without any pain or discomfort. At the time of the examination, the dysphagia, bloody sputum, and pain due to motion of the tongue had all disappeared, and, according to the patient, the cervical swelling was diminishing.

Further intended study of the case by laryngoscopy and roentgenography was prevented by failure of the patient, who lives out of the city, to report subsequently. March 6, information indirectiy obtained revealed only that the patient was still hoarse.

The fact that this patient was treating the external swelling with liniments for a week prior to calling on professional aid. which would not have been sought had she not been frightened by occasional speechlessness, renders it strongly probable that fractures of the greater cornu of the hyoid bone sequential to muscular action are more frequent than shown by the literature, and warrants the belief that cases presenting as many symptoms as the one just narrated, not to mention cases manifesting more vague and fewer symptoms, occur and run their course without recourse to the physician or surgeon.

The treatment was expectant, the patient being ordered to refrain from use of the voice, and to secure approximate immobility of the head and neck by means of wearing a high, stiff collar.

1304 Colwell Street.

\section{A CASE OF BOTHRIOCEPHALUS LATUS INFECTION}

\author{
J. J. Singer, M.D., St. Louis \\ Assistant in Medicine, Washington University
}

Cases of Bothriocephalus latus infection are so rare in this country that it seems advisable to report all cases as they present themselves, if only to awaken interest and stimulate the search for others.

In $1904 \mathrm{Edsal}^{1}$ said that American literature does not provide a sufficient number of cases to indicate that the parasite is by any means common; but the authentic cases that he has found, together with a few reliable cases reported to him in private communications, led him to believe that those whose practices or hospital services brought them in touch with foreigners, especially Russians, Finns, and Swedes, are most likely to meet this parasite.

Many practitioners are content to make a diagnosis of "tapeworms," without identifying the species. This accounts for the small number of known cases.

At any rate, the Bothriocephalus latus is comparatively rare in this country, only twenty-two cases having been reported in America up to 1904. Since then there have been only eight other cases that I could find in the literature on the subject. Only one of these cases ${ }^{2}$ was in an American-born boy, of Finnish parentage. This boy had never left the state of Minnesota. In all probability native foci may develop in the United States through the agency of our foreign-born element, and therefore it is necessary to report and follow up all cases if we wish to keep this parasite from getting a foothold in this country.

Nichorson believes that the probable reason that practically no cases develop in this country is our method of preparing fish for food; the larvae being destroyed in cooking. He states that he has frequently found the larvae of the Bothriocephalus latus in fish caught in the Great Lakes. Huber reports seven cases, seen in as many years, all of which were in Polish patients.

The case I wish to report presents nothing especially new. The specimen of the bothriocephalus is excellent.

The patient, Mrs. I. C., living in St. Louis, a native of Vilna, Russia, called at my office Jan. 23, 1915. She complained of indefinite pains in the abdomen, especially in the left side, lower quadrant. There was no fever or vomiting;

1. Edsal: Am. Med., 1904, viii, 1087.

2. This case was reported by Nichorson in 1906. 